\title{
Posterior Mitral Leaflet Left Atrial Anomalous Chord in the Absence of Surgical Mitral Regurgitation
}

Julie W. Martin, M.D., ${ }^{*}$ and David S. Bach, M.D. $\dagger$

*Division of Cardiology, Department of Internal Medicine, Kaiser Permanente San Diego Medical Center, San Diego, California; and $\uparrow$ The Division of Cardiology, Department of Internal Medicine, University of Michigan, Ann Arbor, Michigan

(Echocardiography 2014;31:E227)

Key words: aberrant mitral valve chord, mitral insufficiency

Aberrant left atrial (LA) mitral valve chords are rare causes of mitral regurgitation (MR). ${ }^{1}$ An aberrant chord on the posterior mitral valve leaflet (PMVL) has not been reported. Transesophageal echocardiogram demonstrated a chord-like structure from the LA to the middle (P2) scallop of the PMVL, causing focal P2 prolapse without

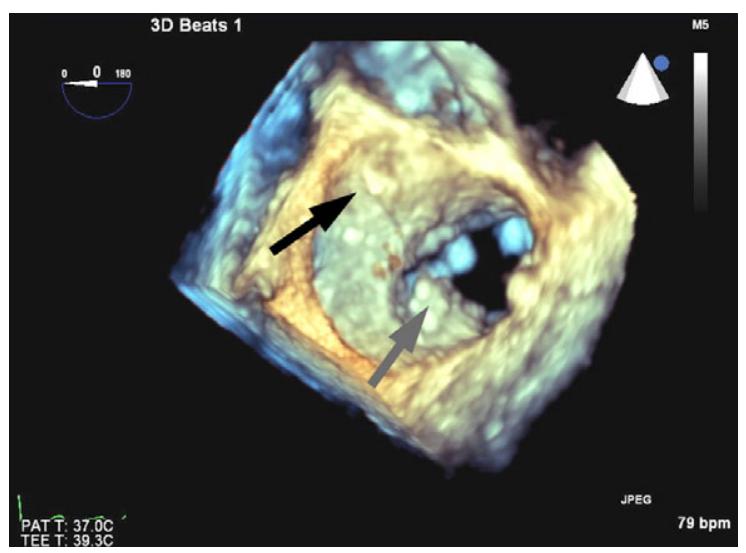

Figure 1. Three-dimensional transesophageal echocardiogram still frame of the mitral valve. There is a papillary musclelike structure (black arrow) within the left atrium. An aberrant chord extends from the left atrium to the middle P2 scallop (grey arrow) of the mitral valve. typical myxomatous degeneration (movie clip S1). Three-dimensional images revealed that this chord terminated onto a papillary muscle-like structure at the LA (Fig. 1). There was only mild MR.

Focal mitral leaflet prolapse without typical myxomatous change may herald the presence of an aberrant chord. Early recognition may alter surveillance needs.

Acknowledgment: We thank Bahram Khadivi, M.D., Kathy Bridge, R.D.C.S., Yasmin Ferreyra, R.D.C.S, and Michael Trenkle, R.D.C.S, for their invaluable assistance with image acquisition and processing.

\section{Reference}

1. Khan $H$, Chaubey $S$, Kenny $C A$, et al: A rare case of an aberrant anterior mitral valve chord resulting in severe mitral regurgitation. JSCR 2011;5:1-3.

\section{Supporting Information}

Additional Supporting Information may be found in the online version of this article:

Movie clip S1. Mid-esophageal view of the mitral valve demonstrating the aberrant chord (arrow). Ao = aorta; LA = left atrium; LV = left ventricle.

Address for correspondence and reprint requests: Julie W. Martin, M.D., Division of Cardiology, Kaiser Permanente San Diego Medical Center, 4647 Zion Avenue, San Diego, CA 92120. Fax: 619-528-6767;

E-mail: Julie.w.martin@kp.org 